\title{
Lidil
}

Revue de linguistique et de didactique des langues

$36 \mid 2007$

Échanges exolingues via internet et appropriation des langues-cultures

\section{Acquisition de compétences interculturelles}

Interactions orales et écrites en tridem en ligne

Isabelle Audras et Thierry Chanier

\section{OpenEdition}

\section{Journals}

Édition électronique

URL : http://journals.openedition.org/lidil/2383

DOI : $10.4000 /$ lidil.2383

ISSN : 1960-6052

Éditeur

UGA Éditions/Université Grenoble Alpes

Édition imprimée

Date de publication : 1 décembre 2007

Pagination : 23-42

ISBN : 978-2-84310-108-3

ISSN : $1146-6480$

Référence électronique

Isabelle Audras et Thierry Chanier, « Acquisition de compétences interculturelles », Lidil [En ligne],

36 | 2007, mis en ligne le 30 juin 2009, consulté le 01 mai 2019. URL : http://journals.openedition.org/ lidil/2383 ; DOI : 10.4000/lidil.2383

Ce document a été généré automatiquement le 1 mai 2019.

(c) Lidil 


\section{Acquisition de compétences interculturelles}

Interactions orales et écrites en tridem en ligne

Isabelle Audras et Thierry Chanier

Dans cet article, nous nous intéressons aux échanges exolingues en groupe en ligne, dans leurs dimensions de prise de risque ou d'engagement qui révèlent l'authenticité et l'existence de l'acte de rencontre de l'autre, acte que nous cherchons à rapprocher de l'acquisition d'une compétence interculturelle. Nous partons d'une étude de cas d'une formation en ligne, qui bénéficie de deux espaces de communication distincts pour l'écrit (blogue) et l'oral (plate-forme audio-synchrone). Nous allons reconstruire la genèse des relations entre apprenants, initialement regroupés en tridems - chacun venant d'une des trois universités partenaires de ce projet de formation. Nous tenterons d'identifier des critères permettant de déceler une réelle vie en ligne, l'idée étant d'associer certains de ces éléments de vie à la manifestation de signes de compétences interculturelles du type de celles apparaissant dans le modèle de Byram.

2 Le propos est structuré en cinq parties : la première donne le cadre théorique; dans la suivante, nous présentons la formation en ligne et l'environnement technologique associé ; puis, dans les troisième et quatrième parties, nous établissons des hypothèses de critères de vie commune en analysant un tridem, hypothèses que nous étoffons par d'autres exemples de tridems dans une cinquième partie.

\section{Formation en langue étrangère en ligne et acquisition d'une compétence interculturelle}

\section{Échanges exolingues en groupes en ligne : utiliser le multimodal pour rencontrer l'autre}

Le développement des réseaux de communications synchrones et asynchrones avec leur entrelacement de modes écrits ou oraux (Chanier \& Vetter, 2006), couplé à l'utilisation de 
collecticiels, qui offrent de riches espaces de travail collaboratif, ouvre naturellement des perspectives aux formateurs de langues, celui de mettre des groupes endolingues dans une situation de correspondance exolingue en croisant langue source et cible. Une situation d'échanges exolingues en groupe en ligne (EEGL) est une situation dans laquelle des groupes d'apprenants résidant dans des pays différents sont impliqués dans des tâches de travail à visée interculturelle en groupe à distance, tâches intégrant des échanges exolingues. Nous privilégions ici les tâches de nature collaborative.

Toute situation d'apprentissage collaboratif, qu'il ait pour finalité les langues ou non, comporte l'éventualité d'un certain nombre de prises de risque pour l'apprenant. En télécollaboration, la cohésion du groupe d'apprentissage ne se construit pas en cherchant à tout prix à éviter les différends. Au contraire, l'occurrence de tels évènements est un signe normal d'engagement des participants. Cet engagement, relié aux deux autres composantes que sont la communication et la coordination, constitue la base de la dynamique de la collaboration, comme l'expliquent les auteurs Henri et Lundgren-Cayrol en présentant leur modèle $(2001: 42)$ :

L'apprentissage collaboratif est une démarche active par laquelle l'apprenant travaille à la construction de ses connaissances. Le formateur y joue le rôle de facilitateur des apprentissages alors que le groupe y participe comme source d'information, comme agent de motivation, comme moyen d'entraide et de soutien mutuel et comme lieu privilégié d'interaction pour la construction collective des connaissances. La démarche collaborative reconnait le caractère individuel et réflexif de l'apprentissage de même que son ancrage social en le raccrochant aux interactions de groupe. [...] L'apprenant s'engage à travailler avec les membres du groupe en vue de la réalisation du but commun tout en conciliant ses intérêts et objectifs personnels.

5 L'occurrence d'évènements correspondant à des prises de risque est d'autant plus probable que nous cherchons ici à constituer à distance des groupes en rassemblant des personnes de langues et cultures différentes. S'engager dans la voie de la collaboration dans les situations EEGL implique de fixer un objectif de produit de travail collectif et d'accorder une attention particulière à l'engagement des acteurs qui se décline en appartenance, cohésion, productivité.

\section{Compétence interculturelle et locuteur interculturel}

6 Les interactions à teneur interculturelle observées lors de formations à distance en langue, quand elles existent, se résument souvent à des échanges d'informations sur la culture-cible (O'Dowd \& Ritter, 2006). Nous y voyons rarement la dimension de prise de risque ou d'engagement qui, pour nous, fait l'authenticité et l'existence de l'acte de rencontrer l'autre, et qui est une des marques d'acquisition d'une compétence interculturelle.

7 La situation d'échanges exolingues en groupe en ligne impose d'emblée l'autre et, à travers la mise en rapport de (au moins) deux groupes et institutions, chacune avec sa légitimité, la présence de plusieurs cultures. L'objectif de développement de la compétence interculturelle, déjà présent en situation de tandem (Lewis 2003), se trouve ainsi posé avec force. Beaucoup de chercheurs citent Byram (1997) pour préciser les constituants de cette compétence, ou plutôt «savoirs » (ce mot étant, pour l'auteur anglophone, le correspondant de competence en anglais). Ces savoirs se déclinent suivant 5 axes : savoir être, où s'apprécie la posture (attitude) d'ouverture et de réflexion sur les 
deux cultures; savoirs (knowledge) dans un sens qui englobe les contenus transmissifs cités précédemment avec une ouverture sur la connaissance des processus de communication dans l'autre culture; savoir comprendre et savoir apprendre-et-faire qui mobilisent les habiletés (skills) à comprendre les documents ou évènements de l'autre culture, à acquérir de nouvelles connaissances à ce propos et à agir de façon appropriée en situation de communication exolingue ; savoir s'engager qui fait appel à des aptitudes d'évaluation critique (critical cultural awareness) des deux cultures et d'engagement à négocier des compromis pour soi-même ou avec les autres, compromis qui dépassent le simple accord sur des références aux normes des cultures de base, pour viser la construction de nouveaux objets acceptables par les différents partis, situés entre les cultures, autrement dit « interculturels ».

8 L'occurrence de conflits lors d'échanges exolingues en groupe en ligne, que nous appellerons dans ce cas «incidents critiques » (Jackson, 2003), a été caractérisée par O'Dowd et Ritter (2006) comme une preuve d'échec de la formation. L'usage de ce mot traduit en fait la tension entre deux enjeux, le social (faire lien, ne pas mettre la relation en péril) et l'enjeu formatif.

9 Nous postulons ici que l'échange exolingue en groupe en ligne peut être l'opportunité d'une authentique expérience de vie interculturelle et collaborative. Nous nous intéressons à révéler les postures du locuteur interculturel qui témoignent d'une vie commune en ligne, où apparaissent démarche personnelle vers l'autre, engagement, ou construction de connaissances visant la découverte de l'autre et de soi. Nous chercherons dans cet article à mettre en évidence les indicateurs d'une telle vie en ligne dans le contexte d'une formation tripartite, à deux langues cibles, alternant espaces de communication à l'oral et à l'écrit.

\section{Présentation de la formation Tridem}

10 La formation à distance Tridem s'est déroulée sur dix semaines, d'octobre à décembre 2005 d'après le scénario suivant. Les apprenants étaient initialement regroupés par trois, en tridems, et venaient chacun d'une des trois institutions partenaires du projet : Carnegie Mellon University (États-Unis, ÉU), The Open University (Royaume-Uni, RU) et l'université de Franche-Comté (France, $\mathrm{Fr}$ ). La formation avait deux langues-cibles : l'anglais pour les francophones et le français pour les anglophones. Au début de la formation, 10 tridems ont été constitués. Au cours de la formation, des fusions de tridems ont eu lieu pour pallier le manque d'activité de certains membres, chaque nouveau tridem préservant l'équilibre entre les trois institutions. On notera, au départ, une disparité importante dans les compétences en L2 entre les groupes, avec des niveaux intermédiaire à avancé au Royaume-Uni et en France, mais débutant pour les apprenants états-uniens.

11 Les apprenants disposaient de deux environnements technologiques de travail distincts: le blogue pour les interactions à l'écrit en asynchrone et la plateforme audio-graphique Lyceum (Chanier et al., 2006) pour les interactions à l'oral en synchrone. Chaque tridem a un blogue dédié. Seuls les membres du tridem et un tuteur y ont les droits d'auteur (les autres participants à la formation ne pouvant que lire et poster des commentaires). Un blogue commun sert de liaison entre tous les participants (tout le monde y est auteur). Quant à Lyceum, trois types de sessions sont possibles: des sessions «programmées » 
autour des activités du scénario, animées par un tuteur, réunissant les membres de plusieurs blogues (les membres d'un même tridem pouvant avoir été dispersés dans des plages horaires de sessions Lyceum différentes en fonction de leur disponibilité ou afin d'encourager les rencontres inter-tridems); des sessions « café » également animées par un tuteur, ouvertes à tous sans sujet prédéfini ; enfin des sessions « libres », non tutorées, à l'initiative des membres d'un même tridem.

Le tuteur était un enseignant de langue, mais pas forcément spécialiste dans les deux langues. L'objectif pédagogique était d'interagir à l'oral et à l'écrit en utilisant sa langue maternelle et la langue cible en vue de construire un écrit dans la langue cible. La thématique de ces interactions était centrée sur la prise de contact et la découverte de l'autre, à travers des activités de la vie quotidienne.

\section{Quels indicateurs pour observer la vie du tridem ?}

13 Nous postulons que le tridem va permettre des interactions à l'écrit et à l'oral dans la langue-cible et la langue-source. Nous cherchons à révéler les interactions qui mettent en scène une rencontre avec l'autre, dans laquelle les locuteurs ont conscience de construire à plusieurs un discours. Cependant, en formation à distance, nous savons que les groupes formés au départ ne sont pas forcément viables, et dans certains cas, il ne s'y passe rien. Le tuteur en ligne se sert généralement d'indications quantitatives pour déceler l'activité du groupe, prévenir l'anomie. Le tableau 1 explicite par tridem le nombre de messages postés dans les blogues et celui de séances Lyceum libres. De façon évidente, les chiffres concernant les blogues 3, 6 et 9 témoignent d'une vie de groupe à l'écrit et à l'oral.

\begin{tabular}{|l|c|c|}
\hline & $\begin{array}{c}\text { Nombre } \\
\text { messages }\end{array}$ & $\begin{array}{c}\text { Nombre séances } \\
\text { Lyceum 'libres' }\end{array}$ \\
\hline Blogue 1 & 26 & 0 \\
\hline Blogue 2 & 19 & 1 \\
\hline Blogue 3 & 81 & 3 \\
\hline Blogue 4 & 50 & 4 \\
\hline Blogue 5 & 28 & 1 \\
\hline Blogue 6 & 76 & 5 \\
\hline Blogue 7 & 22 & 1 \\
\hline Blogue 8 & 15 & 0 \\
\hline Blogue 9 & 101 & 6 \\
\hline Blogue 10 & 6 & 0 \\
\hline
\end{tabular}


Tableau 1- Participation dans les blogues et nombre de séances Lyceum libres

\begin{abstract}
interactions entre membres d'un tridem ont eu lieu, ni si ces interactions ont donné lieu à de véritables rencontres (et non à des séries de monologues dans un même blogue). Il faut donc essayer de mettre en évidence des critères d'observation, non quantitatifs, de vie de tridem. Par exemple des phénomènes de coréférence, d'étayage, d'une modalité à une autre, peuvent, s'ils se répètent lors du déroulement du scénario pédagogique, être de bons indices révélant une relation privilégiée intragroupe. Ces critères d'observation nous aideront, dans un second temps, à caractériser les compétences du locuteur interculturel au sein de cet échange exolingue en groupe en ligne.
\end{abstract}

\title{
Une histoire de vie commune
}

15 Nous allons tout d'abord nous intéresser à décrire et analyser le déroulement de l'échange exolingue dans le blogue 2 car, comme le montre le tableau 1, il fait partie de ceux qui ont produit le moins de messages dans leur blogue. Cela signifie-t-il pour autant que le groupe à trois n'a pas fonctionné, qu'il n'y a pas eu de véritable interaction entre ses membres? En reconstituant le parcours à trois dans le blogue et dans Lyceum, nous dégagerons au contraire l'histoire d'une vraie rencontre.

$\mathrm{Au}$ début de la formation, les trois participants ${ }^{2}, \mathrm{Ch}(\mathrm{Fr}), \mathrm{Ni}(\mathrm{RU})$ et An (ÉU), sont inscrits comme auteurs dans le blogue 2. Deux seulement investissent cet espace de production. Ch poste un message pour se présenter et remplit, ainsi qu'An, la partie «profil » allouée par le logiciel Blogger@ à chaque utilisateur (lieu d'habitation, livres, films, musiques préférés, etc.). Vient le temps de la première session plénière sur Lyceum qui regroupe $\mathrm{Ch}$ et An, aux côtés de 5 autres participants provenant d'autres tridems (2 Français, 1 Étasunien et 1 Britannique) et d'un tuteur. Sur l'invitation du tuteur, An se présente en faisant explicitement référence à ce qu'il a renseigné dans son profil de blogue. À la fin de la séance, au moment de se séparer, Ch invite tous les participants de la session Lyceum dans son blogue :

Rendez-vous dans mon blog, team 2.

[tridem2, blogue, Ch, 17/10]

Ch et An ont bien participé ensemble à cette séance Lyceum, mais aucune trace verbale ne peut laisser supposer qu'ils étaient dans le même tridem. De son côté, Ni a participé à une autre séance Lyceum, programmée à un horaire différent. De retour dans son blogue $2, \mathrm{Ni}$ exprime son ennui et son sentiment d'isolement : il envoie un message d'appel aux autres membres de son blogue. Le tuteur lui répond et l'informe explicitement que Ch et An sont ses partenaires de tridem. $\mathrm{Ni}$ adresse alors un message de bienvenue à $\mathrm{Ch}$ et $\mathrm{An}$. Ce message, auquel An répondra par deux autres, marque la naissance du tridem :

Bonjour Ch et An, comme vous aurez constaté du blog, grâce à [prénom tuteur], je viens de découvrir que je suis dans un tridem avec vous. Excellent! Ch, I think your English in your blog contribution is very impressive. [tridem2, blogue, Ni, 22/10]

Dans ce message, nous pouvons d'ailleurs remarquer que la partie du message directement adressée à Ch est une remarque positive.

19 L'étape suivante du scénario pédagogique prévoit une nouvelle série de séances Lyceum programmées. An et $\mathrm{Ch}$ se retrouvent à nouveau dans la même session. Ch y fait une

Lidil, 36 | 2007 
blague, en montrant une photo du château de Versailles et en le présentant comme sa maison de campagne. An répond à cette blague sur un même ton d'humour :

Your landmaker is good?

[lyceum2, Lyceum, An, 24/10]

À cette manifestation orale, de ce que l'on peut assimiler à une marque de connivence entre les deux, succède un échange dans le clavardage de Lyceum, échange dans lequel $\mathrm{Ch}$ et le tuteur apportent des éléments d'information complémentaires à An afin de l'aider dans son discours oral. Cet échange peut être assimilé à un étayage.

21 Cette séance Lyceum est suivie d'une période de communication asynchrone dans le blogue, doublée d'une séance Lyceum libre dans laquelle se retrouvent, pour la première fois dans un échange oral, les trois membres du tridem. Un fil du discours se construit alors sur le thème du sport avec des marques de coréférences dans les messages des trois participants, dont un message correspondant au compte-rendu de leur séance Lyceum libre.

\section{Diouplay Ruby}

[tridem2, blogue, Ch, 08/11]

Diouplay Ruby

[tridem2, blogue, An, 13/11]

Puis vient la troisième série de sessions Lyceum programmées. Ch est absent. An est donc le seul membre du tridem présent sur cette plage horaire. Dans toutes les sessions programmées antérieures, An n'avait parlé que sur invitation explicite du tuteur. Cette fois, il prend spontanément la parole. Cette augmentation de prise de risque à l'oral est remarquable, d'autant qu'elle a lieu en dehors de l'espace du tridem. De façon comparable, durant cette étape de la formation, Ch se risque, pour la première fois, à intervenir à l'écrit dans un espace hors tridem, le blogue commun, en y initiant un jeu de devinettes.

Enfin, lors de la dernière séance Lyceum programmée, séance de bilan et d'évaluation de la formation, Ch reprend à l'oral ce qu'il considère être les points forts de la formation, après les avoir déjà écrits dans un billet du blogue 2 :

ce que j'ai le plus apprécié durant ce travail dans le blog tridem $2: 1$. le caractère multilingue et multiculturel des échanges, 2 . un fort courant de sympathie entre les participants, 3. le plaisir d'écrire dans une langue étrangère. [tridem2, blogue, $\mathrm{Ch}$, $12 / 12]$

24 An exprimera également, lors d'un entretien post-formation mené par les chercheurs, la joie de la rencontre, qu'il qualifie de « friendship », avec ses deux partenaires de tridem.

\section{Vers des critères de vie du tridem}

\section{Proposition : quels critères révèlent une vie commune en ligne ?}

Suite à la description de cette vie du tridem 2, nous pouvons proposer une liste de critères nécessaires à la construction d'une histoire commune en ligne.

- Le constat de constitution du tridem doit être verbalisé et approuvé par une partie des membres du tridem d'origine ou du tridem reconstitué. Cette prise de conscience peut nécessiter une intervention extérieure (tuteur). Ce constat marque la véritable naissance du tridem, au-delà du simple regroupement prescrit par les formateurs. 

La vie du tridem est ponctuée d'une série de rencontres à l'écrit et à l'oral entre au moins deux membres du tridem. Mais pour arriver au stade de développement d'un véritable tridem, regroupant les trois partis et ressenti comme tel par chacun, il est nécessaire que des rencontres à trois aient lieu (séances Lyceum libres, par exemple), rencontres qui s'appuient sur les échanges précédents (ainsi qu'en témoignent des phénomènes de coréférences aux fils du discours antérieurs).

28 - Les échanges internes au tridem aident à la prise de risque à l'oral et à l'écrit hors du cadre du tridem (phénomène apparu aussi dans le tridem 6 , exemple de vie de tridem suivant, mais non développé ici).

- Un incident critique peut s'avérer positif dans la rencontre de l'autre.

30 tridem 6 a pour éléments actifs Mi (ÉU) et $\mathrm{Br}(\mathrm{Fr})$, avant sa fusion avec le tridem 5 d'où arrive Go (RU). Ju (RU) participe aux mêmes sessions Lyceum que $\mathrm{Br}$ et Mi, mais est membre du tridem 9. Nous essaierons, pour chaque occurrence de critère, de rapprocher la situation décrite de l'une des compétences de Byram.

\section{« Bienvenue dans le tridem »}

31 Dans le blogue 6, la prise de conscience plurielle de l'existence du tridem survient après la première session Lyceum programmée. Br poste son activité, et Mi la sienne. C'est le premier message de $\mathrm{Mi}$ (22 octobre). Br lui répond en lui souhaitant la bienvenue et en lui proposant un rendez-vous à deux dans Lyceum. Cette attitude d'ouverture vers l'autre semble correspondre à la compétence « savoir être » de Byram :

Bonjour Mi ! Je suis contente de voir que tu fais partie du Tridem 6. J'ai également lu la présentation de ta maison avec beaucoup d'intérêt. [...] J'ai beaucoup d'autres choses à te demander mais je crois que ça serait plus facile en discutant sur Lyceum. Penses-tu que nous pourrions planifier une séance dans la semaine en dehors de la séance du mardi ? Dis moi ce que tu en penses ! Br. [tridem6, blogue, $\mathrm{Br}, 22 / 10$ ]

Plus tard, au moment de la fusion des tridems 5 et 6 , Go va également clairement annoncer son arrivée. En sollicitant la reconnaissance des autres, il témoigne de la compétence de « savoir-faire » de Byram :

Toc, toc. Pardon de vous déranger. Je suis un des voisins du Tridem 5 et là-bas il est très calme alors puisqu'on nous dit qu'ici ça bouge autrement bien je suis venu jeter un coup d'oeil. J'espère que ça va et que l'invitation que j'ai reçue par personne interposée a quand même reçu votre aval. [tridem6, blogue, Go, 11/11]

\section{L'histoire se construit au clavier et au micro}

Qu'en est-il des échanges écrits et oraux? Si nous reprenons le duo formé par $\mathrm{Br}$ et $\mathrm{Mi}$, après une première séance libre sur Lyceum (le 25 octobre), nous pouvons remarquer, dans les messages postés à la suite dans le blogue, que chacune a un intérêt propre susceptible de conduire vers des directions différentes. Mi souhaite que Br l'aide dans ses productions en français :

$\mathrm{Br}$, can you correct my French?

[tridem6, blogue, Mi, 26/10] 
Et Br, faisant preuve à nouveau de la compétence de "savoir être », souhaite davantage ouvrir le débat sur d'autres sujets, plus marqués au niveau culturel que ceux présents dans le scénario pédagogique, tout en acceptant de corriger Mi :

the idea [...] to write down [dans Lyceum] some words or expressions in French and English worked pretty well [...]. Back to our subject (expression in French : « pour en revenir à nos moutons »), here are some pictures that describe me best! (laughter). Anyway, have a "spooky» halloween [...]. We could talk about this topic : « Toussaint versus Halloween »... [tridem6, blogue, $\mathrm{Br}, 30 / 10$ ]

$\mathrm{Br}$, parallèlement à cette construction de vie en tandem avec Mi, est entrée en contact écrit avec Ju, depuis la deuxième session Lyceum programmée. Celui-ci a écrit un commentaire à un message de $\mathrm{Br}$, en reprenant l'expression qu'elle venait de lui expliquer: "pour en revenir à nos moutons ». Par ce réemploi, Ju fait preuve de la compétence « savoir comprendre » de Byram :

Pour en revenir à nos moutons. I was really encouraged to see the cellar and cycle park [...]

[tridem6, blogue, Ju, commentaire laissé au message de $\mathrm{Br}$ du 30/10]

Cette connivence à l'écrit marque la distinction des deux rencontres de $\mathrm{Br}$, l'une avec $\mathrm{Mi}$ et l'autre avec Ju. Br réitérera à Mi sa proposition d'ouverture dans un autre message à la suite d'une seconde séance libre sur Lyceum :

$\mathrm{Hi}$ Mi, [...] I discovered your picture of an American football player and also saw it as an opportunity to talk about some controversial matters and stereotypes related to American and French football. I've prepared 2 documents [...].We could try to see if they refer to stereotypes or not? What do you think? [tridem6, blogue, Br, 9/11] Cette étape est importante. C'est pourquoi nous avons choisi de la détailler précisément.

Mi exprime son accord, sans pour autant oublier d'insister sur ses demandes en langue:

Oui ! That sounds great!:) I actually prepaired my leasure document and started my football one although sadly there are no more pics yet!:) And I have a short story that is supposed to switch between the passe compose and imparfait that I could really use your help with if you don't mind... I have no idea how to say all this in French... sigh... [tridem6, blogue, Mi, 9/11]

7 À ce moment-là surviennent la fusion avec le tridem 5 et l'arrivée de Go. Mi reconnait explicitement sa présence. Br l'invite aussitôt à participer aux débats :

Bienvenue à vous Go ! [...]. Pour le thème des loisirs, nous avions pensé partager des opinions sur quelques sports nationaux. Rassurez-vous, je ne m'y connais pas plus en sport que vous, mais l'idée au départ est [...], d'organiser un débat dans notre blog sur nos perceptions respectives des sports nationaux, sur le sport et les médias, les clichés associés aux supporters, etc. (c'est ce que nous avions convenu avec Mi lors de notre session Lyceum du 10 novembre) Qu'en pensez-vous et, toi, Mi es-tu toujours d'accord? [tridem6, blogue, $\mathrm{Br}, 13 / 11$ ]

Mi approuve. Go aussi, tout en ne répondant pas directement sur les sports proposés, mais en glissant (dans un mouvement d'évitement des controverses ?) vers celui du jeu de puces. Il montre à l'occasion une compétence de type « savoir s'engager » :

Puisque les sports d'équipe traditionnels ne sont que des exercices de guerre déguisés et qu'actuellement nous avons marre de ce genre de passe-temps, je vous propose que le monde abandonne le foot, le basket et consorts pour mettre au premier plan les Tiddlywinks [le jeu de puces]. [tridem6, blogue, Go, 14/11] 


\section{Un incident critique peut aider à révéler une compétence interculturelle}

En témoignant de sa capacité à s'engager, Ju a également officiellement ouvert la discussion avec $\mathrm{Br}$, qui le présentera ensuite à Go. Une relation à trois (un tridem ?) est née entre membres de deux tridems différents. Ju va s'introduire, en postant des commentaires, dans une discussion dans le blogue 6 autour de la citoyenneté, discussion initiée par un message de Go, dans lequel nous pouvons remarquer une compétence de type « savoir être » :

La citoyenneté veut dire pour moi une simple relation de sécurité : je paye des taxes à un pays défini par le hazard de ma naissance. [...]. Mes partenaires auront-ils une autre interprétation de ce mot? [tridem6, blogue, Go, 22/11]

$\mathrm{Br}$ témoigne de cette rencontre inter-tridems lors de la dernière séance plénière : Suite à ça [évènement critique dans Lyceum], on a voulu une discussion un peu plus durable, et c'est comme ça qu'est née la discussion sur la citoyenneté [...] [Lyceum4, Lyceum, $\mathrm{Br}, 12 / 12]$ Mi n'a pu être la quatrième partenaire de cet échange à trois. Elle s'en explique dans un message qui clôt le fil de discussion :

[...] my thoughts have been very preoccupied with my brother. he was wounded fighting in Iraq and he is not doing so well, [...] I am not in the right frame of mind to answer a political question on how I feel about citizenship when I am losing my brother to this war.

[tridem06, blogue, Mi, 25/11]

La fin de la formation approchant, il est temps pour chaque tridem de se concentrer sur sa production finale collective (suivant le scénario pédagogique). Br et Go accomplissent ce travail dans le tridem 6, sans l'aide de Mi.

\section{Une histoire de vie du tridem 6 : rencontres en tandem et en tridem}

43 La rencontre entre $\mathrm{Br}$, Mi et Go, même si elle s'est arrêtée brutalement du fait d'un évènement personnel, semble avoir porté ses fruits.

Mi a bien accepté l'arrivée de Go dans le tridem 6, alors qu'elle avait déjà construit une relation de tandem avec $\mathrm{Br}$. Lors d'un entretien après la formation, elle notera, sans toutefois le verbaliser de façon explicite, que le groupe formé par Go, Br et elle ne constituait pas un tridem en soi, mais qu'elle a apprécié de pouvoir parler assez 
ouvertement avec Go. Pour elle, il y a eu une relation de tandem entre Br et elle, et pas de relation de tridem avec Go, du fait qu'ils ne se sont jamais retrouvés tous les trois en session libre Lyceum. On remarque ici l'intérêt de travailler avec une structure de tridem en comparaison de celle du tandem : sa viabilité est plus sûre en cas d'absence d'un des interlocuteurs.

\section{Conclusion} ligne offre une situation authentique de vie commune inter-institutionnelle et plurilingue. En effet, ces quelques exemples de (re)construction de vies de tridems montrent des expériences de découverte de l'autre, qui ne se limitent pas à la simple connaissance de son mode d'habitat ou de ses loisirs, mais s'ouvrent sur de vraies rencontres en ligne: va-t-on se comprendre, arriver à se parler, être capable de s'écouter? Les prises de risque à l'oral et à l'écrit, les évènements de coréférence d'un mode à l'autre, les situations d'étayage entre membres d'un même tridem, les incidents critiques, les discussions en aparté, que cet article a révélés, en sont autant de traces. En élargissant le panorama d'outils indicateurs d'existence de groupe, nous avons fait émerger des critères de vie de tridem, que nous avons d'abord posés en hypothèses de travail avant de les proposer pour l'analyse d'autres tridems. Ils ont effectivement révélé des situations de vie commune intéressantes dans d'autres tridems. Les postures des apprenants dans ces situations ont été rapprochées des savoirs de Byram (1997). caractère interculturel, nous souhaitons approfondir notre approche à l'occasion d'une autre expérience de formation, Tridem06, en introduisant un scénario pédagogique entièrement construit sur l'interculturel, au sens évoqué en introduction, c'est-à-dire construit autour de thèmes de société dans lesquels chaque participant est invité à se positionner et regarder la position de l'autre (voir projet Cultura in Mangenot \& Zourou, ce numéro). De ce type de démarche, on peut espérer mesurer le développement de compétences interculturelles et, en les systématisant, aborder la question de leur transférabilité.

«"Être homme, c'est être capable de transfert dans une autre perspective". Cette formule de Paul Ricoeur est sans doute une des meilleures, sinon la meilleure justification possible à l'apprentissage des langues ", écrit M. Abdallah-Pretceille (Abdallah-Pretceille, 1998). Le postulat de transférabilité implique que le gain de compétence interculturelle, acquis dans une formation mettant en rapport deux cultures, puisse être mobilisé et complété dans des formations ultérieures favorisant d'autres rencontres interculturelles. 


\section{BIBLIOGRAPHIE}

\section{Références}

ABDALLAH-PRETCEILLE, M. (1998) : Apprendre une langue, apprendre une culture, apprendre l'altérité, Cahiers pédagogiques, 360, 49-51.

BYRAM, M. (1997) : Teaching and Assessing Intercultural Communicative Competence, Clevedon, Multilingual Matters.

CHANier, T., VeTter, A., BetBeder, M.-L. \& RefFAy, C. (2006) : Retrouver le chemin de la parole en environnement audio-graphique synchrone, Le français dans le monde, 139-150.

CHANIER, T., VETTER, A. (2006) : Multimodalité et expression en langue étrangère dans une plateforme audio-synchrone, Apprentissage des langues et systèmes d'information et de communication (ALSIC), 9 (1), 61-101.

HENRI, F. \& LUNDGREN-CAYROL (2001) : Apprentissage collaborative à distance, Sainte-Foy, Presses de l'université du Québec.

JACKSON, J. (2003) : «Critical incidents across cultures ». Archives du Subject Centre for Languages, Linguistics and Area Studies, CILT, University of Southampton.

LEWIS, T. \& WALKER, L. (dir.) (2003) : Autonomous Language Learning in Tandem, Sheffield, Academy Electronic Publications

O'DOWD R. \& RITTER M. (2006) : Understanding and Working with « Failed communication » in tellecollaborative exchange, Computer Assisted Language Instruction Consortium Journal (CALICO Journal), 23 (3), 623-642.

\section{NOTES}

1. Nous utilisons le service de Blogger $\odot$

2. Pour des raisons de préservation de l'anonymat, les prénoms des participants ont été partout remplacés par des codes à deux lettres.

\section{RÉSUMÉS}

Rares sont les expériences multiculturelles en ligne, en groupes multi-institutionnels et multilingues, qui permettent d'aboutir à l'observation d'attitudes de prise de risque ou d'engagement chez les participants, postures qui pour nous révèlent l'authenticité et l'existence de l'acte de rencontrer l'autre. En 2005, nous avons conçu une formation en ligne mettant en scène des échanges exolingues en groupe, en partant de l'hypothèse que cette rencontre de 
l'autre sera facilitée, d'une part en regroupant les apprenants en tridem - chacun venant d'une des institutions partenaires de cette formation - et, d'autre part, en combinant deux espaces de communication (blogue et plate-forme audio-synchrone). À partir des données recueillies lors de cette expérience, nous reconstruisons la genèse et la vie des tridems. Nous identifions des critères qualitatifs permettant de déceler une réelle vie en ligne, en particulier le développement de fils de discours reliant écrit et oral, l'occurrence d'incidents critiques. Nous associons certains de ces éléments de vie à la manifestation de signes de compétences interculturelles du type de celles apparaissant dans le modèle de Byram.

Interactions with intercultural content in language learning are generally summarized with exchanges of information on a culture-target. We seldom observe a mark of engagement or openness which make the authenticity and the existence of the act to meet somebody, and which is for us the proof of an intercultural competence acquisition. This means to take an important risk but this one is necessary for learning to discover the other and one-self. This learning corresponds to 6 steps by Byram: savoirs, savoir comprendre, savoir apprendre/faire, savoir être, savoir s'engager. We want to observe this steps of intercultural learning, in distance groups exolingues exchanges, that is to say multilingual, multicultural and multi-institutional learners groups. In the distance language learning that we chose to study, there are two different workspaces: one for the writing communication (blog) and one the oral one (Lyceum audiosynchronous platform). Language learners are initially gathered in tridems and come each one of three different universities. We are interesting in observing this situations of common life on line by the help of the 6 steps of Byram. We suppose that this study will help to find observable points for the construction of an intercultural competence on line, in order to be able to think about its transfer.

\section{AUTEURS}

\section{ISABELLE AUDRAS}

Université de Franche-Comté

\section{THIERRY CHANIER}

Université de Franche-Comté 